\title{
Estratégias de Sondagem para Remapeamento Eficiente de Eventos de Roteamento na Internet
}

\author{
Elverton Fazzion $^{1,2}$ Dorgival Guedes $^{1} \quad$ Wagner Meira Jr. $^{1} \quad$ Ítalo Cunha $^{1}$ \\ ${ }^{1}$ Departamento de Ciência da Computação \\ Universidade Federal de Minas Gerais (UFMG) \\ ${ }^{2}$ Departamento de Computação \\ Universidade Federal de São João del-Rei (UFSJ) \\ fazzioneufsj.edu.br \{dorgival, meira, cunha\}edcc.ufmg.br
}

\begin{abstract}
Path changes caused by events such as traffic engineering, changing peering agreements, and link failure impact many routes in the Internet. Topology monitoring platforms perform periodic traceroute measurements toward a large number of destinations. This approach, however, is inadequate to precisely identify the extent of paths involved in the event. For example, a link failure can be restored before all routes are measured. In this work we present measurement strategies that minimize the probing cost for identifying paths impacted by a routing event. Our results show that it is possible to efficiently identify the set of paths impacted by a routing event. Our results also indicate that, when integrated to a state-of-the-art path change tracking system, our strategies more than double the number of path changes detected.
\end{abstract}

Resumo. Mudanças de caminho causadas por eventos como engenharia de tráfego, alteração de parcerias de troca de tráfego, ou falhas de enlace impactam vários caminhos na Internet. Plataformas de monitoramento topológico realizam medições periódicas usando traceroute para um grande número de destinos. Esta abordagem, porém, é inadequada para identificar precisamente a extensão do impacto de eventos de roteamento. Por exemplo, uma falha de enlace pode ser restaurada antes que todas as rotas sejam medidas. Neste trabalho apresentamos estratégias de medição que minimizam o custo de sondagem para identificar caminhos impactados por um evento de roteamento. Nossos resultados mostram que é possível identificar o conjunto de caminhos impactados por um evento de forma eficiente. Nossos resultados indicam ainda que, quando integradas a um sistema estado-da-arte de rastreamento de mudanças de caminhos, nossas estratégias mais que dobram o número de mudanças detectadas.

\section{Introdução}

A infraestrutura da Internet cresceu exponencialmente na última década. Atualmente, milhões de roteadores, conectados por bilhões de caminhos, são responsáveis pelo roteamento de dados entre as diversas partes do globo. Eventos de roteamento, como reconfiguração de roteadores ou falhas de enlace, podem impactar diretamente o desempenho da rede, gerando grandes prejuízos financeiros como o caso recente em que a China Telecom repassou, acidentalmente, anúncios de rotas indevidos feitos por um provedor africano, deixando vários serviços do Google inacessíveis para diversos 
usuários [Goodin 2018]. Entender o impacto de eventos de roteamento na Internet é essencial tanto para auxiliar operadores de redes a resolver problemas rapidamente quanto para ajudar pesquisadores a desenvolverem técnicas que melhorem a rede.

Identificar os diversos caminhos afetados por eventos de roteamento em uma topologia é desafiador. A maioria dos sistemas e plataformas que medem a infraestrutura subjacente ainda fazem medições periódicas de traceroute para atualizar caminhos na topologia [Kompella et al. 2007, Katz-Bassett et al. 2012, Claffy et al. 2009, Cunha et al. 2016]. Embora essa estratégia possa funcionar para identificar problemas em redes pequenas, ela não é adequada para medir o impacto de grandes eventos na Internet. O grande número de sondas requeridas pelo traceroute para medir um grande número de caminhos, combinado com baixas taxas de sondagem impostas a tráfego de monitoramento para não afetar o tráfego da rede, implica em longos períodos de medição que resultam na coleta de informações incompletas ou inconsistentes para eventos de curta duração [Cunha et al. 2011, Ravaioli et al. 2015]. Além disso, a abordagem periódica ainda evita que observemos, com precisão, a extensão de um evento de roteamento pois outros eventos de roteamentos podem acontecer antes de remapearmos todos os caminhos impactados pelo primeiro evento.

Neste artigo, apresentamos estratégias para identificar, de forma eficiente e rápida, possíveis caminhos em uma topologia que foram impactados por um evento de roteamento. Dada uma mudança em um caminho entre uma origem e um destino (zona de mudança), nossas estratégias identificam quais outros caminhos na topologia intersectam a zona de mudança (caminhos intersectantes). Nossas estratégias estimam quais caminhos intersectantes possuem a maior probabilidade de serem impactados pelo evento de roteamento e verificando de forma eficiente quais caminhos efetivamente mudaram.

Para avaliar nossas estratégias, instrumentamos o DTRACK [Cunha et al. 2014], uma ferramenta de monitoramento de mudanças de roteamento na internet do estadoda-arte, para coletar dados que capturam eventos de roteamento. Avaliamos diferentes abordagens para cada passo no processo de verificação de mudanças, em particular abordagens utilizando algoritmos de aprendizado de máquina. Mostramos que as estratégias propostas - apesar de simples, intuitivas, computacionalmente baratas, e sem necessidade de treinamento prévio-são tão eficazes quanto técnicas mais complexas ou abordagens usando aprendizagem de máquina. Nossa avaliação indica que nossas estratégias, se integradas ao DTRACK, permitiriam um aumento significativo na verificação de mudanças em caminhos intersectantes. Neste artigo fazemos as seguintes contribuições:

- Mostramos que podemos verificar se um caminho intersectante mudou (ou não) usando uma única sonda.

- Mostramos que o tamanho da interseção de um caminho com uma zona de mudança é correlacionado com a probabilidade do caminho ser afetado pelo evento. Enumeramos também propriedades dispensáveis para identificar mudanças.

- Propomos e avaliamos diferentes estratégias de sondagem para detecção de caminhos impactados por eventos de roteamento.

Nossas estratégias permitem caracterização de eventos de roteamento com precisão antes impossível, melhorando o entendimento da dinâmica de rotas na Internet por operadores de rede e pesquisadores. Além disso, mostramos que esta precisão pode ser obtida utilizando estratégias simples e sem aumento significativo do custo de sondagem. 


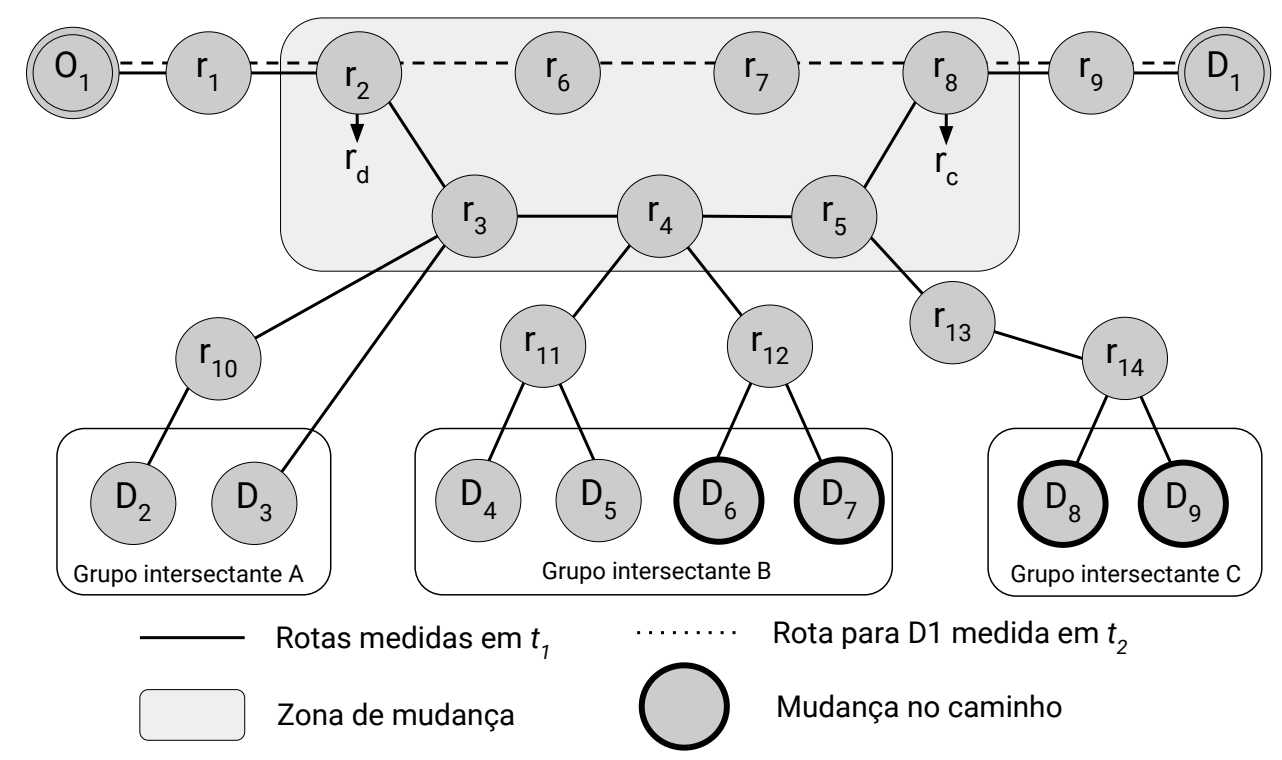

Figura 1. Exemplo de mudança de caminho e definições.

\section{Definições e Fundamentos}

Seguindo definições na literatura, um caminho virtual (caminho) é a conectividade entre uma origem e um destino na Internet. Em um dado momento $t$, um caminho virtual entre a origem $o_{i}$ e o destino $d_{j}$, denominado $C_{o_{i} d_{j}}$, é instanciado por uma rota $C_{o_{i} d_{j}}(t)$. Omitimos a origem no subscrito pois ela é fixa (o ponto de medição) e omitimos o subscrito por completo quando o destino estiver claro pelo contexto.

Devido a mudanças de roteamento, um caminho pode ser visto como um processo contínuo que muda de uma rota $C(t)$ a outra ao longo do tempo. Uma rota é formada por uma sequência de interfaces enumeradas a partir da origem. A identificação da rota ativa num instante $t$ é realizada por meio de medição traceroute. Neste artigo assumimos que medições são realizadas com Paris traceroute e usam o mesmo identificador de fluxo [Augustin et al. 2011]) para evitar balanceamento de carga em rotas na Internet. Na figura 1 , o caminho de $o_{1}$ para $d_{1}$ é instanciado pela rota $C\left(t_{1}\right)=\left[o_{1}, r_{1}, r_{2}, r_{3}, r_{4}, r_{5}, r_{8}, r_{9}, d_{1}\right]$ no instante $t_{1}$ (linha sólida) e pela rota $C\left(t_{2}\right)=$ $\left[o_{1}, r_{1}, r_{2}, r_{6}, r_{7}, r_{8}, r_{9}, d_{1}\right]$ no instante $t_{2}$ (linha pontilhada).

Definimos uma zona de mudança comparando duas medições de rotas consecutivas, $C\left(t_{i-1}\right)$ e $C\left(t_{i}\right)$. Uma zona de mudança é uma sequência contígua de saltos na rota $C\left(t_{i-1}\right)$ que não existe na rota $C\left(t_{i}\right)$. Uma zona de mudança possui um ponto de divergência definido como a interface imediatamente anterior à sequência, e um ponto de convergência definido como a interface imediatamente posterior à sequência. (Essas interfaces estão presentes em ambas as rotas.) Denotamos uma zona de mudança entre as rotas $C\left(t_{i-1}\right)$ e $C\left(t_{i}\right)$ por $Z\left(C, t_{i}\right)$. A figura 1 mostra um exemplo com uma zona de mudança $Z\left(C_{d_{1}}, t_{2}\right)=\left\{r_{2}, r_{3}, r_{4}, r_{5}, r_{8}\right\}$ onde o ponto de divergência é $r_{2}$ e o ponto de convergência é $r_{8}$.

Uma zona de mudança $Z\left(C_{d_{i}}, t_{i}\right)$ pode ser intersectada por rotas de outros caminhos $C_{d_{j}}, i \neq j$. Mais precisamente, todo caminho $C_{d_{j}}$ cuja última rota conhecida $C_{d_{j}}\left(t_{j}\right)$ possui algum salto contido na zona de mudança $Z\left(C_{d_{i}}, t_{i}\right), t_{j}<t_{i}$, é denominado um $c a$ minho intersectante. Nós agrupamos caminhos intersectantes com interseções idênticas em grupos intersectantes. Na figura 1 , as rotas $C_{d_{2}}\left(t_{1}\right)$ e $C_{d_{3}}\left(t_{1}\right)$ formam um grupo inter- 
sectante que intersecta dois salto da zona de mudança, enquanto $C_{d_{8}}\left(t_{1}\right)$ e $C_{d_{9}}\left(t_{1}\right)$ formam outro grupo intersectante que intersecta quatro saltos da zona de mudança.

\section{Medição e Tratamento de Rotas}

Nesta seção descrevemos os dados coletados para orientar o desenvolvimento e sustentar a avaliação de nossas estratégias de sondagem.

\subsection{Ferramenta de Medição}

O DTRACK [Cunha et al. 2014] é um sistema que rastreia mudanças em um conjunto de caminhos na Internet. Após uma fase inicial de medição dos caminhos monitorados, o DTRACK inicia um processo de detecção de mudanças de roteamento. O processo de detecção de mudanças permite detectar mais mudanças, com latência de detecção menor, do que medições periódicas com traceroute. Quando uma mudança é detectada em algum caminho, o DTRACK remapeia o caminho usando o traceroute e reinicia o processo de detecção de mudanças.

Nós modificamos o DTRACK para coletar dados que nos permitissem caracterizar estratégias de remapeamento de eventos de roteamento. Após remapear um caminho onde uma mudança foi detectada, nossa versão modificada calcula a zona de mudança e imediatamente remapeia todos os caminhos intersectantes. Comparada à abordagem comum de realizar medições periódicas usando traceroute, nossas medições reduzem a latência para detecção do evento de roteamento (em função de usarmos o DTRACK) e a latência para medir rotas potencialmente impactadas pelo evento (em função do remapeamento imediado dos caminhos intersectantes). Nosso objetivo é medir os caminhos intersectantes, potencialmente impactados pelo evento de roteamento, antes do evento terminar ou antes da ocorrência de outros eventos relacionados.

Essa abordagem não garante que iremos remapear todos os caminhos impactados pelo evento de roteamento. Em particular, caminhos que não intersectam a zona de mudança ou que não são monitorados podem ser impactados pelo evento e não serão remapeados. Esta limitação, porém, é intrínseca à impossibilidade de realizar medições de todos os dispositivos na Internet e a limitações de taxa de sondagem. Esta limitação se aplica a qualquer abordagem de medição. Para uma taxa de sondagem fixa, argumentamos que a abordagem utilizada minimiza a latência de detecção e remapeamento de eventos de roteamento.

\subsection{Conjunto de Dados}

Nós implantamos o DTRACK estendido em 15 nós do PlanetLab distribuídos nos cinco continentes. Geramos listas de destinos representativas utilizando uma hitlist com mais de 1,2 milhões de endereços IP distintos (únicos por /24) e altamente disponíveis, cobrindo 34.224 sistemas autônomos (AS) diferentes. Para cada nó do PlanetLab, nós construímos uma lista distinta com 5.000 endereços IP. Para cada nó PlanetLab, escolhemos aleatoriamente um AS e depois escolhemos aleatoriamente até dez endereços IPs dentro do AS, repetindo este processo até totalizar 5.000 endereços IP. Para garantir a cobertura de caminhos com grande volume de tráfego, adicionamos aos destinos os endereços IP associados aos 500 sites mais acessados segundo o Alexa. ${ }^{1}$ Coletamos medições entre

\footnotetext{
${ }^{1}$ https://www.alexa.com/topsites
} 


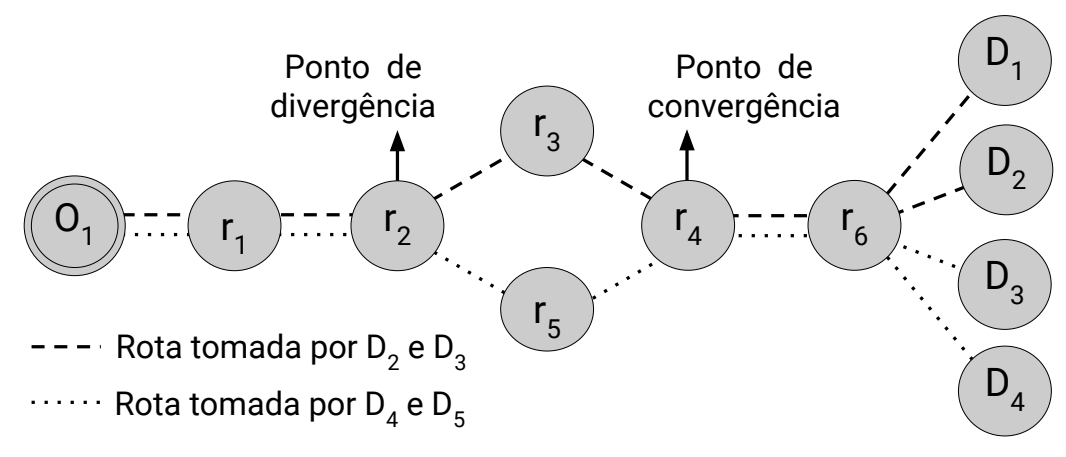

Figura 2. Exemplo de identificação de balanceador de carga por destino.

23 e 28 de novembro de 2018 (6 dias completos). No período, o DTRACK detectou 1.1 milhões de eventos de roteamento, e realizamos 3,4 milhões de medições traceroute.

\subsection{Balanceadores de Carga por Destino}

Interfaces que fazem balanceamento de carga (e.g., usando ECMP) podem causar erros em medições de traceroute [Augustin et al. 2011]. Balanceamento de carga também pode ser incorretamente interpretado como mudanças de caminho [Cunha et al. 2011]. O DTRACK mantém uma base de dados de todos as interfaces que fazem balanceamento de carga por fluxo (i.e., utilizando portas de origem e destino ou o checksum do cabeçalho ICMP) para evitar interpretações incorretas. Além disso, realizamos todas nossas medições usando a implementação do Paris traceroute no scamper [Luckie 2010].

Porém, como neste trabalho calculamos interseções entre caminhos para destinos diferentes, nossa análise é impactada por interfaces que fazem balanceamento de carga por destino (i.e., utilizando os endereços IP de origem e destino). Nesta seção descrevemos como identificamos interfaces que fazem balanceamento de carga por destino para evitar interpretações incorretas de mudanças de caminho.

Rotas passando por um balanceador de carga formam diamantes (figura 2), onde o balanceador é chamado também de ponto de divergência, que distribui pacotes entre diferentes interfaces no salto seguinte, e onde uma interface subsequente é o ponto de convergência, onde todas as rotas convergem. Nós identificamos como balanceadores de carga por destino todos as interfaces em nosso conjunto de dados que criam um diamante (i.e., com pontos de divergência e convergência definidos) e onde cada interface dentro do diamante é atravessado por pelo menos três caminhos com destinos diferentes. Na nossa base de dados identificamos balanceadores de carga por destino em 31.1\% dos caminhos, taxa similar a resultados prévios encontrados na literatura [Augustin et al. 2011].

A figura 2 mostra quatro rotas para os caminhos $C_{d_{1}}, C_{d_{2}}, C_{d_{3}}$ e $C_{d_{4}}$ passando por um diamante formado pelas interfaces $r_{2}, r_{3}, r_{4}$ e $r_{5}$. Os caminhos $C_{d_{1}}$ e $C_{d_{2}}$ passam pela interface $r_{3}$ e os caminhos $C_{d_{3}}$ e $C_{d_{4}}$ passam pela interface $r_{5}$. Neste exemplo, a interface $r_{2}$ seria classificada como um balanceador de carga por destino se exigíssemos 2 caminhos por cada interface no diamante.

\section{Verificando Rotas Impactadas com Baixo Custo de Sondagem}

Dada uma zona de mudança $Z\left(C_{d_{i}}, t_{i}\right)$ e um caminho intersectante $C_{d_{j}}$, uma abordagem simples para identificar se $C_{d_{j}}$ foi impactado pelo evento de roteamento é executar o traceroute para medir a rota atual $C_{d_{j}}\left(t_{j}\right)$ e comparar com a anterior $C_{d_{j}}\left(t_{j-1}\right), t_{j-1}<t_{i}<t_{j}$, para verificar se houve mudança. Entretanto, essa estratégia é ineficiente pois poucos 


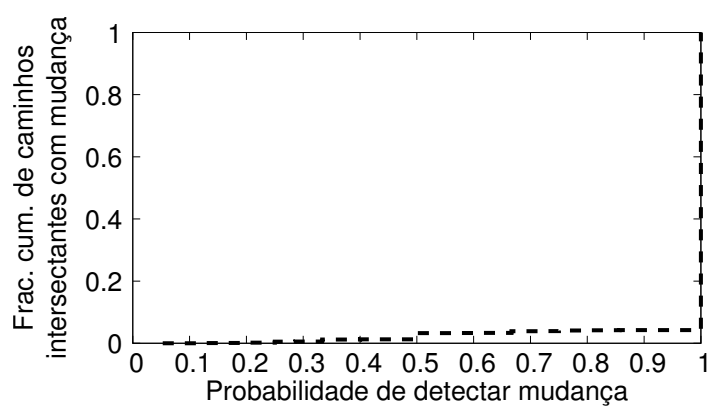

Figura 3. Probabilidade de detectar mudanças num caminho sondando uma sua interseção com a zona de mudança.

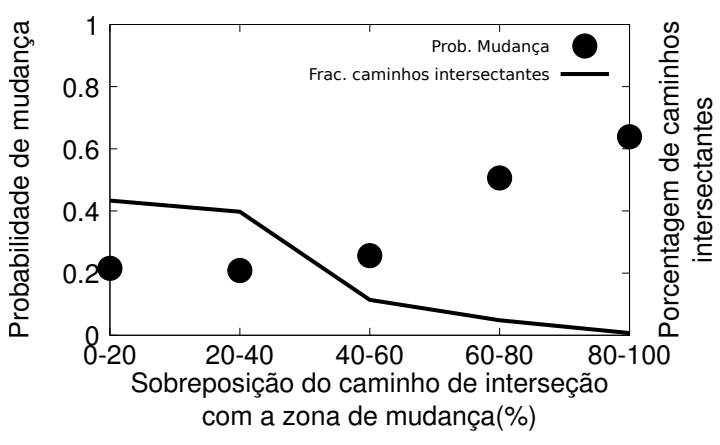

Figura 4. Probabilidade de um caminho intersectante mudar dado o tamanho da interseção com zona de mudança.

caminhos mudam e dezenas de sondas são necessárias para executar cada traceroute, levando a desperdício de sondas [Cunha et al. 2014]. Este desperdício de sondas impacta a capacidade de sistemas reais em manter a topologia da rede atualizada e em capturar a extensão de um evento de roteamento.

Estudamos como verificar mudanças num caminho intersectante $C_{d_{j}}$ de forma eficiente. Quando um caminho intersectante $C_{d_{j}}$ muda, i.e., existe $Z\left(C_{d_{j}}, t_{j}\right)$, podemos verificar a mudança enviando uma única sonda para qualquer uma de suas interfaces que pertencem a $Z\left(C_{d_{j}}, t_{j}\right)$. Com base nessa observação, tentamos encontrar um ponto de sondagem que nos permite verificar uma mudança em $C_{d_{j}}$ de forma confiável. Mais precisamente, dadas uma zona de mudança $Z\left(C_{d_{i}}, t_{i}\right)$ e a última medição de um caminho intersectante $C_{d_{j}}\left(t_{j-1}\right)$, queremos enviar uma sonda no instante $t_{j}$ que identifica uma mudança em $C_{d_{j}}$, se existir.

Identificamos que sondar o ponto de divergência de $Z\left(C_{d_{i}}, t_{i}\right)$ raramente verifica uma mudança em um caminho intersectante $C_{d_{j}}$ por que $78.05 \%$ das mudanças em caminhos intersectantes possuem o mesmo ponto de divergência. Porém, identificamos que para a maioria dos caminhos intersectantes $C_{d_{j}}$ com mudança, todas as demais interfaces que intersectam a zona de mudança são impactadas, i.e., $\left\{C_{d_{j}}\left(t_{j-1}\right) \cap Z\left(C_{d_{i}}, t_{i}\right)\right\} \subset$ $Z\left(C_{d_{j}}, t_{j}\right)$. A figura 3 mostra a distribuição, para os caminhos intersectantes $C_{d_{j}}$ que mudaram, da fração de interfaces do caminho intersectante que intersectam $Z\left(C_{d_{i}}, t_{i}\right)$ e permitem verificar a mudança em $C_{d_{j}}$, i.e., $\left|Z\left(C_{d_{j}}, t_{j}\right) \cap Z\left(C_{d_{i}}, t_{i}\right)\right| \div\left|C_{d_{j}}\left(t_{j-1}\right) \cap Z\left(C_{d_{i}}, t_{i}\right)\right|$. Observamos que para mais de $96 \%$ dos caminhos intersectantes $C_{d_{j}}$ que mudaram, todas suas interfaces em $Z\left(C_{d_{i}}, t_{i}\right)$ permitem verificar a mudança em $C_{d_{j}}$. O resultado na figura 3 implica que podemos enviar uma única sonda para qualquer interface em $Z\left(C_{d_{i}}, t_{i}\right)$ para verificar se $C_{d_{j}}$ mudou de forma confiável, reduzindo drasticamente o número de sondas necessárias comparada com a execução de um traceroute.

\section{Ordenando Caminhos Intersectantes por Probabilidade de Mudança ${ }^{2}$}

A Figura 4 mostra a probabilidade de um caminho intersectante mudar dada a fração de interfaces da zona de mudança no caminho (círculos, eixo $y_{1}$ ). Agrupamos os caminhos intersectantes pelo tamanho da interseção com a zona de mudança (eixo $x$ ). Notamos que quanto maior o tamanho da interseção de um caminho com a zona de mudança,

\footnotetext{
${ }^{2}$ Uma versão preliminar da análise das seções 4 e 5 apareceu em um resumo estendido publicado na seção de pôsteres do SIGCOMM 2016 [Fazzion et al. 2016].
} 


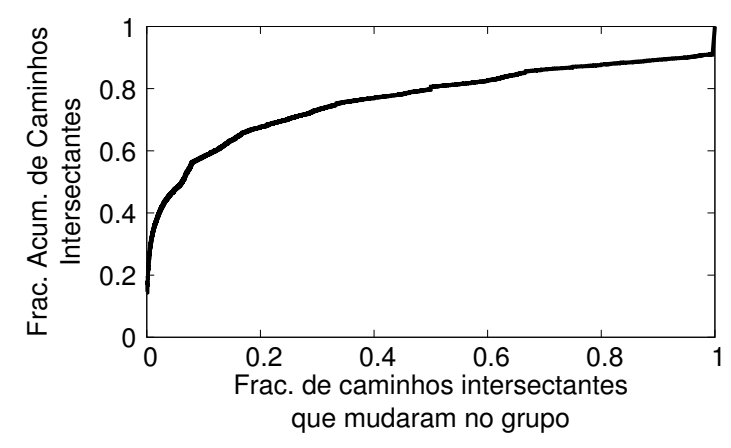

Figura 5. Distribuição de caminhos por probabilidade de mudança no grupo.

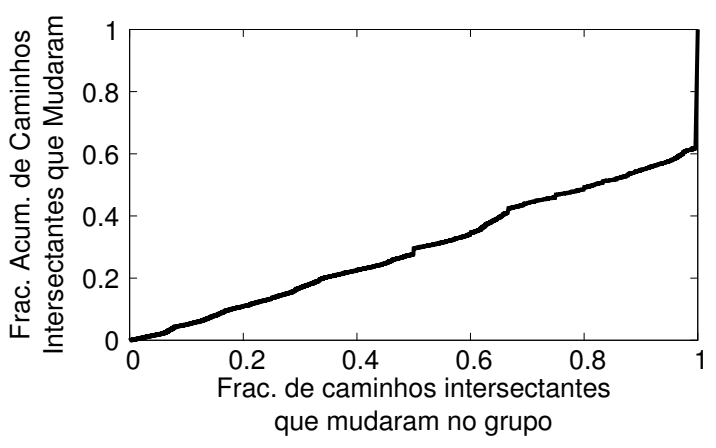

Figura 6. Distribuição das mudanças por probabilidade de mudança no grupo.

maior a probabilidade do caminho intersectante mudar. Este resultado indica que no exemplo da figura 1 , os caminhos intersectantes $C_{d_{8}}$ e $C_{d_{9}}$ (80\% de interseção) têm maior probabilidade de mudar que os caminhos intersectantes $C_{d_{2}}$ e $C_{d_{3}}$ (40\% de interseção). A figura 4 também mostra que a maior parte dos caminhos possuem interseções pequenas com a zona de mudança (linha, eixo $y_{2}$ ).

Avaliamos o comportamento de caminhos intersectantes pertencentes a um mesmo grupo. A figura 5 mostra a distribuição da fração de caminhos que mudam em um grupo de interseção. Observamos que $30 \%$ dos caminhos fazem parte de grupos onde nenhum outro caminho mudou (lado esquerdo do gráfico) e que $10 \%$ dos caminhos fazem parte de grupos onde todos os caminhos mudaram (parte direita do gráfico). De forma mais geral, $75 \%$ dos caminhos fazem parte de grupos "quase homogêneos" onde $90 \%$ dos caminhos não mudam ou $90 \%$ dos caminhos mudam $(x \leq 0.1$ e $x \geq 0.9)$. Este resultado sugere que a sondagem de um caminho em um grupo de interseção pode nos ajudar a tomar decisões quanto à sondagem de outros caminhos no mesmo grupo. A figura 6 é similar à figura 5, mas considera apenas os caminhos intersectantes que mudaram. Vemos que a maioria dos caminhos intersectantes que mudaram estão concentrados em grupos intersectantes nos quais a maior parte dos caminhos mudam, indicando que podemos considerar a composição de um grupo no processo de verificação de mudanças.

\section{Verificando Mudanças em Cenários com Taxa de Sondagem Limitada}

Sistemas reais de monitoramento de rede controlam a taxa de sondagem para reduzir o impacto de medições sobre o tráfego [Shavitt and Weinsberg 2009, Claffy et al. 2009]. Considerando que um sistema de monitoramento opera com um orçamento de sondagem (e.g., em sondas por segundo), é necessário determinar uma sequência de caminhos intersectantes a sondar de forma a maximizar a utilidade das sondas disponíveis. Neste trabalho, consideramos a utilidade das sondas como a fração do número de mudanças encontradas pelo número de sondas enviadas. Propomos duas abordagens de sondagem baseadas nos resultados anteriores (seções 6.1 e 6.2) e uma abordagem utilizando algoritmos de aprendizado de máquina (seção 6.3). Discutimos um esquema de realocação de sondas entre eventos de roteamento (seção 6.4) e comparamos a efetivadade das técnicas propostas (seção 6.5).

\subsection{Sondagem por Grupo de Interseção}

Motivados pela observação que caminhos em um grupo de interseção frequentemente são impactados de forma idêntica por um evento de roteamento (seção 5), propomos um 
processo de sondagem por grupo. Ao verificar uma mudança em um grupo, consideramos que existe alta probabilidade de outros caminhos do mesmo grupo terem mudado e continuamos sondando outros caminhos do grupo. Dessa forma, o processo de sondagem concentra sondas em um grupo intersectante com muitos caminhos impactados pelo evento de roteamento. Quando sondamos um caminho e verificamos que ele não mudou, consideramos que o grupo pode ter poucos caminhos impactados pelo evento de roteamento, cessamos a sondagem do grupo atual e passamos a sondar o próximo grupo de interseção. Como o tamanho da interseção com a zona de mudança é relacionada à probabilidade de mudança (seção 5), sondamos grupos por ordem decrescente do tamanho da interseção. Dentro de cada grupo sondamos caminhos aleatoriamente. Quando uma verificação de mudança falha no grupo com menor interseção, a técnica retorna ao grupo com maior interseção. Esse processo é repetido até que todos os caminhos intersectantes sejam sondados ou que o orçamento de sondas seja exaurido.

A figura 1 apresenta um exemplo de zona de interseção com oito caminhos intersectantes separados em três grupos $A, B$ e $C$ onde os caminhos $C_{d_{6}}, C_{d_{7}}, C_{d_{8}}$ e $C_{d_{9}}$ mudaram. Suponha que o orçamento de verificação para este evento é de 5 sondas. A sondagem por grupo de interseção inicia sondando pelo grupo $C$ e verifica mudanças nos caminhos $C_{d_{8}}$ e $C_{d_{9}}$ usando 2 sondas. A técnica então sonda o caminho $C_{d_{6}}$ no grupo $B$. Como essa sonda verifica uma mudança, a técnica sonda o caminho $C_{d_{4}}$ ainda no grupo $B$. Como $C_{d_{4}}$ não mudou, a técnica passa para o grupo $A$ e envia a última sonda em $C_{d_{2}}$. Neste exemplo, verificamos 3 de 4 mudanças (75\%) com um orçamento de verificação cobrindo 5 de 8 caminhos intersectantes $(62,5 \%)$.

\subsection{Sondagem por Probabilidade Condicional de Mudança}

Uma limitação da sondagem por grupo de interseção é que a técnica passa a sondar o próximo grupo mesmo quando o grupo corrente tem vários caminhos impactados pelo evento de roteamento. Para solucionar esta limitação propomos uma técnica que calcula a probabilidade condicional de verificar mudanças em um grupo de interseção e sonda sempre caminhos no grupo de interseção com maior probabilidade condicional. Calculamos a probabilidade condicional de verificar uma mudança em um grupo $G$ dados o número de sondas $s_{G}$ enviadas a caminhos no grupo e o número $v_{G}$ de mudanças verificadas usando a lei da probabilidade total:

$$
P\left(\operatorname{mudança}(G) \mid s_{G}, v_{G}\right)=\sum_{G_{i} \in \mathcal{G}_{i}}\left[P\left(\operatorname{mudança~}\left(G_{i}\right) \mid G_{i}, s_{G}, v_{G}\right) \times P\left(G_{i} \mid s_{G}, v_{G}\right)\right],
$$

onde $\mathcal{G}_{i}$ é um subconjunto dos grupos sondados no passado e $P\left(G_{i} \mid s_{G}, v_{G}\right)$ é estimado usando o teorema de Bayes. ${ }^{3} P\left(\operatorname{mudança}\left(G_{i}\right) \mid G_{i}, s, v\right)$ e $P\left(G_{i} \mid s, v\right)$ são calculados assumindo que a fração de caminhos impactados pelo evento de roteamento em $G_{i}$ é dada por $v_{G_{i}} / s_{G_{i}}$. Nossos resultados mostram que quanto maior a razão $v_{G} / s_{G}$, maior a probabilidade de verificar outras mudanças em $G$.

Particionamos os grupos sondados no passado em cinco subgrupos $\mathcal{G}_{i}$, onde o $i$-ésimo subgrupo contém os grupos cujos caminhos intersectam entre $20(i-1)$ a $20 i$ porcento da zona de mudança. Esta abordagem nos permite calcular $P(\operatorname{mudança}(G) \mid 0,0)$ e $P\left(\right.$ mudança $\left.(G) \mid s_{G}, v_{G}\right)$ a partir do subgrupo $\mathcal{G}_{i}$ correspondente ao tamanho da interseção de caminhos em $G$ com a zona de mudança. Desta forma melhoramos a precisão da es- 
timativa da probabilidade de mudança considerando que o tamanho da interseção com a zona de mudança é correlacionada à probabilidade de mudança.

Ilustramos o funcionamento da técnica no exemplo da figura 1 e um orçamento de cinco sondas. A probabilidade de detectar mudanças nos grupos $A, B$ e $C$ é calculada utilizando $\mathcal{G}_{2}, \mathcal{G}_{3}$ e $\mathcal{G}_{5}$, respectivamente. Como $P(\operatorname{mudança}(C) \mid 0,0) \quad>$ $P($ mudança $(B) \mid 0,0)>P($ mudança $(A) \mid 0,0)$, a técnica inicia a sondagem pelo grupo $C$. Após verificar uma mudança, a probabilidade condicional de verificação de mudanças aumenta, i.e., $P($ mudança $(C) \mid 1,1)>P(\operatorname{mudança}(C) \mid 0,0)$, e a técnica envia mais uma sonda ao grupo $C$, verificando mudanças em $C_{d_{8}}$ e $C_{d_{9}}$. A técnica então passa a sondar o grupo $B$, sonda e verifica uma mudança em $C_{d_{6}}$. Como $P(\operatorname{mudança}(B) \mid 1,1)>$ $P(\operatorname{mudança}(B) \mid 0,0)$, a técnica envia uma sonda em $C_{d_{4}}$ e verifica que o caminho não mudou, o que abaixa a probabilidade condicional de mudanças em $B$, i.e., $P($ mudança $(B) \mid 2,1)<P($ mudança $(B) \mid 1,1)$. A próxima sonda será enviada para o grupo $A$ ou $B$ dependendo da probabilidade condicional de detectarmos mudança em cada grupo. Supondo que $P(\operatorname{mudança}(B) \mid 2,1)>P($ mudança $(A) \mid 0,0)$, o algoritmo continuaria sondando o grupo $B$, poderia enviar a última sonda em $C_{d_{7}}$ e verificar todas as 4 mudanças com apenas 5 sondas.

\subsection{Sondagem por Aprendizado de Máquina}

Avaliamos também a aplicabilidade de algoritmos de aprendizado de máquina para determinar qual caminho intersectante deve ser sondado a cada instante. Para treinar os algoritmos de aprendizagem identificamos e calculamos 19 características (features) de caminhos intersectantes. As características podem ser classificadas em três tipos: (i) atributos do caminho intersectante, como o número de ASes na interseção com a zona de mudança e a fração de interseção com a zona de mudança, (ii) atributos da zona de mudança, como o número de ASes envolvidos na zona de mudança e o número de grupos de interseção; e (iii) atributos de grupos de interseção como o número de caminhos intersectantes no grupo.

Avaliamos quatro algoritmos de classificação com aplicabilidade conhecida: RandomForest, SVM, regressão logística e kNN. Treinamos os modelos usando o Weka (v3.8.2) com parâmetros padrões dos algoritmos e validação cruzada com 5-folds. Treinamos os algoritmos de aprendizagem de máquina com 2.056 .772 caminhos intersectantes de 60 mil eventos de roteamento (zonas de mudança). ${ }^{4} \mathrm{O}$ algoritmo com melhor desempenho foi o RandomForest, com acurácia de $91 \%$ e $F_{1}$-score de 0.911 . Omitimos os resultados de nossa análise e consideramos apenas o RandomForest nas seções seguintes.

\subsection{Número de Sondas de Verificação}

Plataformas reais de monitoramento de rotas na Internet trabalham com um orçamento de sondagem. Por exemplo, a plataforma do RIPE Atlas utiliza com um sistema de créditos

\footnotetext{
${ }^{3} \mathrm{O}$ termo $P\left(s_{G}, v_{G} \mid G_{i}\right)$ pode ser estimado usando uma distribuição hipergeométrica, que descreve a probabilidade de $v_{G}$ sucessos (verificações) em $s_{G}$ tentativas (sondagens), sem reposição, de uma população de $\left|G_{i}\right|$ caminhos onde $v_{G_{i}} / s_{G_{i}}$ dos caminhos mudaram.

${ }^{4} \mathrm{~A}$ limitação do treino com 60 mil eventos de roteamento é devida a limitações de tempo de execução e memória disponível no servidor utilizado (16 cores e 82GB de RAM). Porém, nossa análise indica que um conjunto de treino maior não deve melhorar significativamente a precisão dos modelos. Por exemplo, um modelo RandomForest treinado em 16000 eventos de roteamento possui acurácia de 90\%.
} 
que limita a quantidade e taxa de medições que usuários podem realizar, e monitores da plataforma Ark sondam a uma taxa fixa de 100 sondas/segundo.

Considerando estas restrições em cenários onde enviar sondas para verificar mudanças tem baixa probabilidade de sucesso (seção 5, figuras 5 e 6), nossa estratégia de sondagem cessa o processo de verificação para economizar sondas e utilizá-las para verificação de mudanças em eventos futuros. Mais precisamente, nossa estratégia de sondagem tenta manter um saldo de sondas de forma a controlar a probabilidade de escassez de sondas abaixo de um limiar $L_{\text {déficit }}$.

Nós estimamos um limite superior da probabilidade de escassez de sondas fazendo suposições conservadoras. Primeiro, supomos que a distribuição de mudanças entre eventos é a menos uniforme possível: ou todos os caminhos em um evento mudam, ou nenhum caminho em um evento muda. Segundo, nós utilizamos uma estimativa conservadora da probabilidade de mudança dos caminhos, denotada $p_{\text {mudanca. }}$. Se o saldo de sondas for suficiente para verificar mudanças em $k$ eventos de roteamento, a probabilidade de ocorrer escassez de sondas é dada por uma distribuição geométrica:

$$
p_{\text {escassez }}=p_{\text {mudanca }}^{k}\left(1-p_{\text {mudanca }}\right)<L_{\text {déficit }}
$$

Mantemos uma janela deslizante com histórico dos últimos $W$ eventos de remapeamento para estimar $p_{\text {mudanca }}$ e $k$. Calculamos $p_{\text {mudanca }}$ como a fração de sondas enviadas

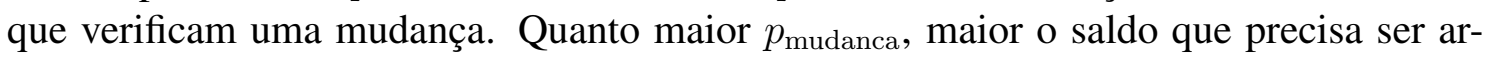
mazenado para cobrir frequentes eventos (futuros) com mudanças. Note que $p_{\text {mudanca }}$ superestima a probabilidade de mudança, pois o processo de sondagem (seções 6.1-6.3) concentra sondas nos grupos com muitas mudanças. Calculamos o valor de $k$ dividindo o saldo corrente de sondas pela quantidade média de sondas que precisamos utilizar para verificar mudanças em um evento onde todos os caminhos mudam (i.e., a diferença entre o número médio de caminhos intersectantes por evento e o orçamento médio de sondagem por evento). Quanto menor a quantidade de sondas que precisamos utilizar do saldo para verificar mudanças em um evento, menor o saldo que precisa ser armazenado.

\subsection{Avaliação das Técnicas de Sondagem}

Nesta seção, comparamos as três técnicas apresentadas (seções 6.1-6.3) em função do orçamento de sondagem. Para fins de exposição, expressamos o orçamento de sondagem em função do número médio de caminhos intersectantes por evento. Mostramos resultados para $W=300$ eventos e $L_{\text {déficit }}=0.1$, mas resultados para outros valores similares resultam em resultados quantitativamente similares.

A figura 7 mostra a utilidade da sondas em função do orçamento de sondagem para as diferentes técnicas, um oráculo que sonda apenas caminhos que mudam e sondagem aleatória sem economia de sondas. A figura 8 mostra a fração de mudanças detectadas em função do orçamento de sondagem para as mesmas abordagens. Como esperado, observamos que a utilidade das sondas diminui e a fração de mudanças detectadas aumenta à medida que o orçamento de sondagem aumenta. O mecanismo de controle da probabilidade de escassez de sondas adapta-se a qualquer orçamento de sondagem e permite às técnicas alocarem sondas de forma eficiente, com ganhos expressivos se comparadas a sondagem aleatória. 


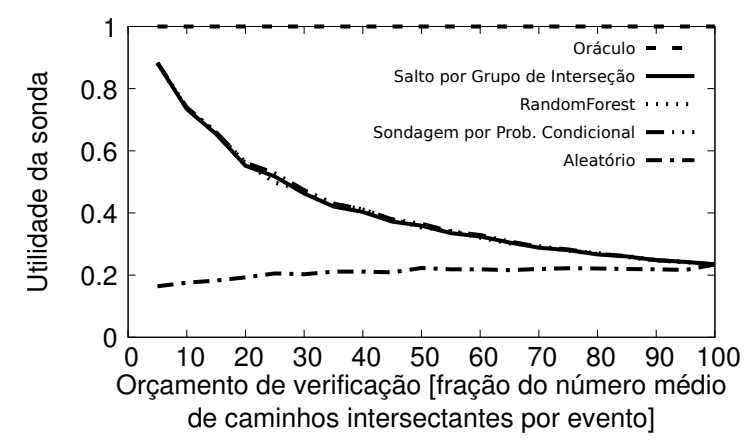

Figura 7. Utilidade da sonda entre as técnicas.

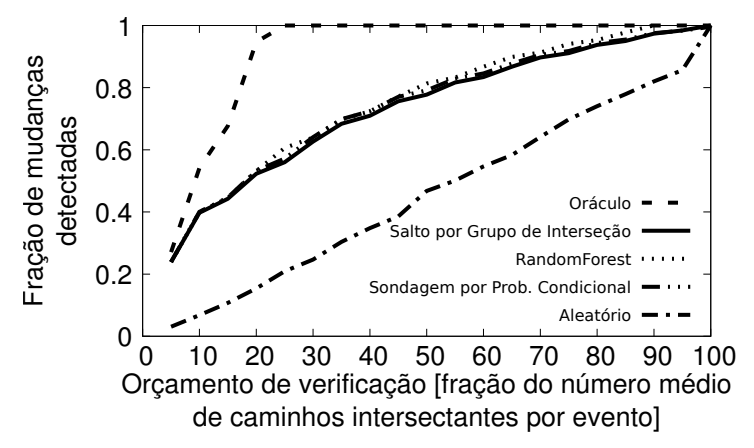

Figura 8. Fração de mudanças detectadas entre as técnicas.

As três técnicas avaliadas possuem desempenho similar. Argumentamos pelo uso da técnica de sondagem por grupo de interseção, que é significativamente mais simples, intuitiva, não mantém estado e não necessita de treinamento. Dado os ganhos marginais das outras duas técnicas quando comparadas à sondagem por grupo de interseção, conjecturamos que a melhoria de desempenho necessitaria de abordagens significativamente mais complexas ou usando fontes de dados complementares.

\subsection{Comparativo com o DTRACK}

Para cenários com baixo orçamento de verificação (e.g., igual a 0.2), nossas técnicas verificam duas vezes mais caminhos intersectantes que mudaram. Esse valor aumenta consideravelmente para cenários com alto orçamento de verificação (e.g., igual a 1), onde a eficiência chega a ser cinco vezes maior. Ou seja, de todos os caminhos intersectantes que mudaram em nossa base, o DTRACK, em sua configuração original, remapearia apenas $20 \%$ deles. Este resultado indica que o remapeamento de mudanças de roteamento causadas por um evento requer um processo de sondagem direcionado como o proposto neste artigo.

Notamos ainda que uma simples extensão do DTRACK que remapeia todos os caminhos intersectantes na tentativa de detectar mudanças aumenta a quantidade de medições necessárias significativamente. Nossas técnicas, se aplicadas ao DTRACK, reduzem o custo de medições para caminhos que não mudam a uma única sonda e permitem verificar a maioria das mudanças mesmo para baixos orçamentos de sondagem (figura 8).

\section{Particionamento de Grupos de Interseção}

Nesta seção avaliamos outras formas de particionar caminhos intersectantes em grupos. Na seção 5 mostramos que caminhos com interseção idêntica em geral são afetados de forma similar por eventos de roteamento. Nesta seção consideramos características adicionais que podem estar correlacionadas com caminhos serem afetados por um evento de roteamento para agrupar caminhos intersectantes.

A intuição por trás das características avaliadas é que eventos de roteamento podem impactar apenas alguns caminhos da Internet, dependendo de fatores, por exemplo, como interface, enlaces e ASes em uma rota. Considere, por exemplo, os caminhos $C_{d_{4}}, C_{d_{5}}, C_{d_{6}}$ e $C_{d_{7}}$ na figura 1 , originalmente no grupo $B$. Neste exemplo, o evento de roteamento afetou rotas passando por $r_{12}$, mas não afetou rotas passando por $r_{11}$. Se subdividirmos o grupo $B$ pela interface visitada imediatamente após a interseção com a zona 
de mudança $\left(r_{11}\right.$ e $r_{12}$ ), formaríamos dois subgrupos homogêneos, o que melhoraria o desempenho da técnica de sondagem por grupos de interseção. Consideramos as seguintes características para subparticionamento de grupos:

Próxima interface. Dada a última interface $r$ pertencente à interseção de um caminho com uma zona de mudança, essa característica representa a interface sucessora de $r$ no caminho intersectante. Na figura 1, a próxima interface no caminho $C_{d_{2}}$ é $r_{10}$.

Próximo AS. Dada o última interface $r$ pertencente à interseção de um caminho com uma zona de mudança, essa característica representa o Sistema Autônomo (AS) da interface sucessora de $r$ no caminho intersectante. Na figura 1, o próximo AS no caminho $C_{d_{2}}$ é o AS de $r_{10}$.

Próximo AS diferente. Dado o AS $x$ da última interface $r$ pertencente à interseção de um caminho com uma zona de mudança, essa característica representa o primeiro AS $y \neq x$ correspondente a alguma interface posterior a $r$ no caminho intersectante. Na figura 1 , supondo que os ASes de $r_{5}, r_{13}$ e $r_{14}$ sejam $x, x$ e $z$, respectivamente, o próximo AS diferente no caminho $C_{d_{8}}$ é $z$ (o AS de $r_{14}$ ).

Próximo roteador de borda. Dado o AS $x$ da última interface $r$ pertencente à interseção de um caminho com uma zona de mudança, essa característica representa a última interface pertencente ao AS $x$ posteria a $r$ no caminho intersectante. Na figura 1, supondo que os ASes de $r_{5}, r_{13}$ e $r_{14}$ sejam $x, x$ e $z$, respectivamente, o próximo roteador de borda no caminho $C_{d_{8}}$ é $r_{13}$.

A figura 9 é similar à figura 5 e mostra a distribuição da fração de caminhos que mudam em um grupo de interseção. Cada linha corresponde a grupos de interseção definidos por caminhos com diferentes características. Observamos que diferentes características têm impacto pequeno nos grupos de interseção, com próximo AS diferente, próxima interface, e próximo roteador de borda resultando em grupos mais homogêneos (maior concentração nas bordas e linhas mais horizontais). Esta maior homogeneidade, porém, é obtida ao custo de grupos de interseção com poucos elementos (não mostrado).

A figura 10 é similar à figura 8 fração de mudanças detectadas em função do orçamento de sondagem para diferentes definições de grupos de interseção quando usamos a técnica de sondagem por grupo de interseção (seção 6.1). O resultado para as outras técnicas de sondagem é quantitativamente similar. Observamos que diferentes definições do grupo de interseção têm impacto quase nulo na quantidade de mudanças detectadas. Apesar das técnicas com grupos mais homogêneos permitirem a identificação de outros caminhos no mesmo grupo que provavelmente mudaram (ou não), estas técnicas também têm grupos menores que limitam a quantidade de caminhos para os quais podemos fazer esta identificação. No pior caso quando o grupo de interseção possui um único caminho que não mudou, a sonda não provê informação útil para o processo de sondagem.

Como as diferentes definições de grupos de interseção resultam em desempenho equivalente para verificação de mudanças, argumentamos novamente pela abordagem mais simples: grupos formados por caminhos com interseção idêntica com a zona de mudança (sem características adicionais avaliadas nesta seção). De forma geral, nossos resultados indicam que a relação de eventos de roteamento e características de caminho, se existir, é complexa e difícil de capturar. 


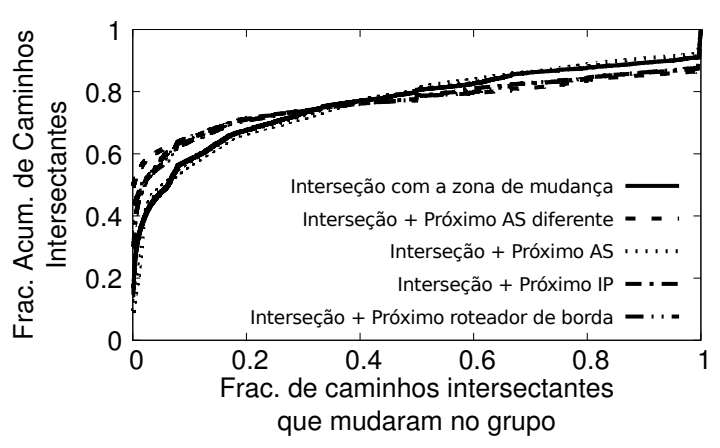

Figura 9. Distribuição de caminhos por probabilidade de mudança para diferentes definições de grupos de interseção.

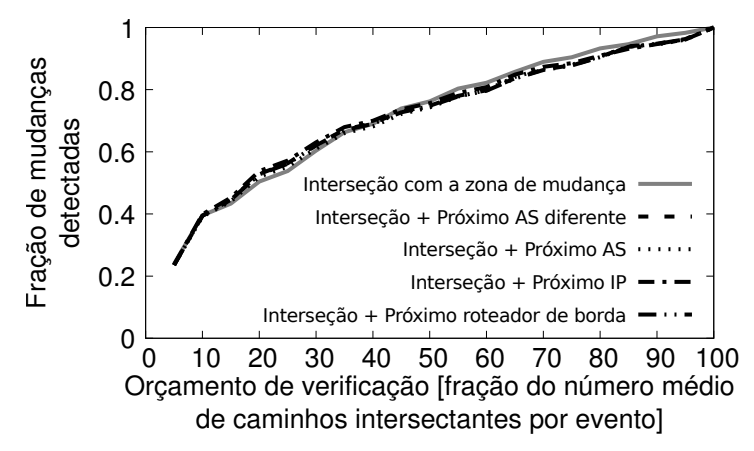

Figura 10. Fração de mudanças detectadas para diferentes definições de grupos de interseção.

\section{Trabalhos Relacionados}

Um desafio em mapeamento topológico é melhorar a cobertura de caminhos. Progresso é feito primariamente pela expansão de plataformas de monitoramento como o Ark, RIPE Atlas e M-Lab. Projetos de pesquisa estendem a cobertura de plataformas de monitoramento, por exemplo, integrando pontos de medição de diferentes plataformas [Levchenko et al. 2017], facilitando utilização programática de pontos de medição [Giotsas et al. 2016] e prevendo rotas a partir de medições existentes [Cunha et al. 2016]. Outro desafio é coletar medições precisas e metadados. Avanços recentes incluem técnicas para identificar balanceadores de carga IPv6 [Almeida et al. 2017], roteadores de borda [Marder et al. 2018] e a localização de roteadores [Giotsas et al. 2015]. Nosso trabalho complementa estes trabalhos reduzindo o custo de medições e permitindo o monitoramento de mais caminhos ou realização de medições mais complexas.

Nosso trabalho é mais similar e complementa outras técnicas que visam reduzir a quantidade de sondas necessárias para coletar medições de roteamento na Internet, por exemplo reduzindo o custo para detecção de mudanças de rota [Cunha et al. 2014], identificação de roteadores que fazem balanceamento de carga [Vermeulen et al. 2018].

\section{Conclusão}

Neste trabalho estudamos como capturar o impacto de eventos de roteamento em caminhos na Internet através da verificação de quais caminhos foram impactados pelo evento com baixo custo de sondagem. Mostramos que uma única sonda é suficiente para verificar mudança em um caminho que intersecta uma zona de mudança. Mostramos que quanto maior a interseção de um caminho com a zona de mudança, maior a probabilidade do caminho ser impactado pelo evento de roteamento. Por fim, propomos e avaliamos diferentes técnicas de sondagem, com diferentes configurações e complexidade, e mostramos que todas são igualmente eficazes. Nossos resultados justificam o uso de abordagens simples e intuitivas. Mais importante, nossos resultados indicam que o relacionamento de eventos de roteamento com mudanças de rota, se existir, é complexo. Como trabalho futuro, pretendemos integrar nossa ferramenta no DTRACK e realizar medições na Internet para caracterizar eventos de roteamento na Internet.

\section{Agradecimentos}

Este trabalho foi parcialmente financiado pela FAPEMIG, CNPq, CAPES e CTIC/RNP. 


\section{Referências}

Almeida, R., Fonseca, O., Fazzion, E., Guedes, D., Meira, W., and Cunha, Í. (2017). A Characterization of Load Balancing on the IPv6 Internet. In Proc. PAM.

Augustin, B., Friedman, T., and Teixeira, R. (2011). Measuring Multipath Routing in the Internet. IEEE/ACM Trans. Netw., 19(3):830-840.

Claffy, K., Hyun, Y., Keys, K., Fomenkov, M., and Krioukov, D. (2009). Internet Mapping: from Art to Science. In Proc. IEEE CATCH.

Cunha, I., Marchetta, P., Calder, M., Chiu, Y.-C., Machado, B. V. A., Pescapè, A., Giotsas, V., Madhyastha, H. V., and Katz-Bassett, E. (2016). Sibyl: A practical internet route oracle. In Proc. USENIX NSDI.

Cunha, I., Teixeira, R., and Diot, C. (2011). Measuring and Characterizing End-to-End Route Dynamics in the Presence of Load Balancing. In Proc. PAM.

Cunha, I., Teixeira, R., Veitch, D., and Diot, C. (2014). DTRACK: A System to Predict and Track Internet Path Changes. IEEE/ACM Trans. Netw., 22(4):1025-1038.

Fazzion, E., Cunha, I., Guedes, D., Meira, Jr., W., Teixeira, R., Veitch, D., and Diot, C. (2016). Efficient remapping of internet routing events. In SIGCOMM (SRC).

Giotsas, V., Dhamdhere, A., and kc claffy (2016). Periscope: Unifying Looking Glass Querying. In Proc. of PAM.

Giotsas, V., Smaragdakis, G., Huffaker, B., Luckie, M., and claffy, k. (2015). Mapping Peering Interconnections to a Facility. In Proc. ACM CoNEXT.

Goodin, D. (2018). Google goes down after major bgp mishap routes traffic through china. Ars Technica.

Katz-Bassett, E., Scott, C., Choffnes, D. R., Cunha, I., Valancius, V., Feamster, N., Madhyastha, H. V., Anderson, T., and Krishnamurthy, A. (2012). LIFEGUARD: Practical Repair of Persistent Route Failures. In Proc. ACM SIGCOMM.

Kompella, R., Yates, J., Greenberg, A., and Snoeren, A. (2007). Detection and Localization of Network Blackholes. In Proc. IEEE INFOCOM.

Levchenko, K., Dhamdhere, A., Huffaker, B., claffy, k., Allman, M., and Paxson, V. (2017). PacketLab: A Universal Measurement Endpoint Interface. In Proc. IMC.

Luckie, M. (2010). Scamper: A scalable and extensible packet prober for active measurement of the internet. In Proc. IMC.

Marder, A., Luckie, M., Dhamdhere, A., Huffaker, B., claffy, k., and Smith, J. M. (2018). Pushing the Boundaries with bdrmapIT: Mapping Router Ownership at Internet Scale. In Proc. IMC.

Ravaioli, R., Urvoy-Keller, G., and Barakat, C. (2015). Characterizing icmp rate limitation on routers. In Proc. IEEE Conference on Communications.

Shavitt, Y. and Weinsberg, U. (2009). Quantifying the Importance of Vantage Points Distribution in Internet Topology Measurements. In Proc. IEEE INFOCOM.

Vermeulen, K., Strowes, S. D., Fourmaux, O., and Friedman, T. (2018). Multilevel mdalite paris traceroute. CoRR. 